\title{
DOMESTIC WASTE MANAGEMENT AS A MEANS FOR LIFE QUALITY IMPROVEMENT AND ENVIRONMENTAL PROTECTION IN THE CITY OF KARDITSA: CITIZENS' VIEWPOINT
}

\author{
P. KARANIKOLA \& S. TAMPAKIS \\ Department of Forestry and Management of the Environment and Natural Resources, \\ Democritus University of Thrace, Greece.
}

\begin{abstract}
This project refers to the investigation of opinions of Karditsa municipality citizens with respect to waste management and cleanness in their town. About $57.7 \%$ of the citizens state that they are unaware of the municipality's cleanness regulation, while with respect to information about waste management, $31.2 \%$ claim to be sufficiently informed, and $31.4 \%$ have poor information. However, most citizens find the cleanness services provided to be satisfactory $(58.4 \%)$. Citizens who evaluate the cleanness services as being 'satisfactory to very satisfactory' live at a distance of up to $50 \mathrm{~m}$ from the waste containers, and state that the containers are emptied more than 3 times a week, while those who claim to be 'disappointed to very disappointed' are located at a distance more than $50 \mathrm{~m}$ away from the containers and claim that waste is collected 1-3 times a week. Almost half of the citizens are aware of containers being moved, and usually dispose of waste in the morning and evening, while the vast majority $(80.5 \%)$ denied leaving waste outside the containers when these are empty. Finally, around half of the citizens are unaware of the amount they pay through municipal taxes for cleanness and waste disposal.
\end{abstract}

Keywords: citizens'viewpoint, environmental protection, Karditsa city, waste management.

\section{INTRODUCTION}

In the past, financial evolution and urbanism together with over-consumption of products resulted in the constant waste growth. The quality of wastes was encumbered proportionally and the produced polluting loads, by the decomposition processes, were transmitted into the ground, air and underground water [1]. Many activities focusing in certain areas result in the concentration of waste by-products that can reach such high levels of stress and develop social reactions [2]. Therefore, proper management of domestic wastes is compulsory, if we as a society seek after the environmental protection and quality improvement in urban areas.

The aim of the present project is to form a wider viewpoint in relation to the attitude of the citizens of Karditsa towards their domestic waste management. Furthermore, we are interested in system management and how it is conceived by the citizens; more specifically, whether the citizens are informed about cleanness regulations and waste management of their city or not; their personal evaluation on cleanness services and how they are connected to their estimation, with respect to the distance from the waste containers and the frequency of the wastes' collection; at what time of the day they dispose their waste into the containers; whether they dispose their litter beside the containers that are empty at that time; whether they notice that the containers are being moved and whether they litter 'unconsciously' streets or communal areas. Furthermore, we are interested in whether they are aware of the amount they pay, through the public fees, for cleanness and waste collection.

\section{RESEARCH METHODOLOGY}

The research was carried out with the application of personal interviews. An interview is the best way for statistical data collection [1]. The municipality of Karditsa formed the research area. Simple 
random sampling was applied due to its simplicity and the fact that it requires less possible knowledge about the population compared to any other methods [3-7].

'Population' under investigation (41,411 citizens) is the total of the households of the municipality of Karditsa. Simple random sampling presupposes the existence of a full catalog (sampling frame) of the population data without deficiencies or reiterations [8]. The applied sampling frame was the consumers' lists of domestic electricity as $100 \%$ of the households in the research area have electricity. Household use forms a classic case for using a group of people instead of persons as a sampling unit. This is because in some cases it is more convenient and less expensive [5]. The procedure for the member selection (from the random selected household) was therefore organized in this way to avoid selecting the same member (i.e. the leader or wife etc.) [8]. The estimation of the population proportion which is also the objective estimation of the real population proportion $p$, is rendered from the relationship:

$$
\bar{p}=\frac{\sum_{i=1}^{n}\left(p_{i}\right)}{n} .
$$

The typical error of the population proportion estimation, $s_{\bar{p}}$, (eqn (2)), without the correction of the finite population (eqn (3)), due to the fact that both the fraction and the period of trustfulness are small, is rendered from the following relationships:

$$
\begin{gathered}
s_{\bar{p}}=\sqrt{\frac{\bar{p}(1-\bar{p})}{n-1},} \\
p=\bar{p} \pm t \cdot s_{\bar{p}},
\end{gathered}
$$

where $t$ is the rate of student's distribution for the probability $(1-\alpha)=95 \%$ and $n-1$ degrees of freedom.

The sampling size was estimated according to the types of simple random sampling $[3,5,7]$. Although simple random sampling without replacement was applied, the correction of finite population can easily be ignored, because the sampling's size is smaller when compared to the $N$ population size $[3,9]$. Since variables refer to proportions, the determination of the total size of the sample is given by the following relationship:

$$
n=\frac{t^{2} \bar{p}(1-\bar{p})}{e^{2}}=\frac{1.96^{2} \cdot 0.5 \cdot(1-0.5)}{0.05^{2}}=384.16 \cong 385,
$$

where $t$ is the rate of Student's distribution for the probability $(1-\alpha)=95 \%$ and $n-1$ degrees of freedom. Due to the large size of the sample ( $n$ is larger than 50), rate $t$ is taken from the probability tables of normal distribution for the desired probability. In fact, for the $95 \%$ probability, rate $t$ is 1.96 [5]. $p=$ proportion estimation; $e=$ maximum acceptable difference between sampling medium and unknown population medium. We accept that it is 0.05 , i.e. $5 \%$.

In order to estimate the sample size, we carried out a pre-sampling for 50 people. From this, the actual population proportion and sample size was estimated for each variable. If the size of the estimated samples is contiguous and the total size is within the financial possibilities of the sampling, then the maximum size is selected for the sample ( 385 citizens). This way, the most varied variable is estimated according to the desired precision, whereas the rest are estimated with greater precision than originally determined [5]. 
The households of the sample are then traced with accuracy (that is full name and address) with the aid of random numbers taken from relevant tables. Personal interviews with a randomly selected member of the family were carried out in the selected households. In case, the house was empty, or when there was a refuse for an interview, we tried two more efforts to get their viewpoints. If this was impossible, then we continued with the same procedure for the selection of new sampling units. The collection data was carried out in 2003 and for the analysis, the Statistical Package SPSS was applied. For the variables 'rendered cleanness services', 'distance from the waste containers' and 'collection of the waste containers per week' frequency analysis, with more than two criteria, was carried out. The hierarchical log-linear analysis was applied.

Prior to the initiation of the log-linear analysis (in particular the hierarchical), it is intentional to examine the size of the expected frequencies in the crossing table [10]. Large number of the expected frequencies (higher than 20\%) with rate lower than 5, but not lower than 1, run the risk of leading to the power loss of the applied analysis [11]. This examination is carried out by control of doublesided crossing tables through the SPSS program $[12,13]$.

The classes of variables were grouped, in order to satisfy the above-mentioned conditions. Our data are classified in accordance with three criteria and express frequencies. The assumption $H_{\mathrm{o}}$ is:

$$
H_{\mathrm{o}} \text { : three criteria are fully independent from each other. }
$$

It is unlikely for this assumption to be accepted, but the analysis will allow the precise level of various interrelations and will be included in a model that expresses the data interrelations [13].

Statistic controls of optimum adjustment are used for the estimation of the corresponding degree between the model and the data. Statistical significance shows that the examined model does not represent the observed frequencies perfectly, whereas the statistical non-significance means that the examined model is adjusted to the observed frequencies, respectively. $\chi^{2}$ test is applied [14].

Log-linear analysis forms a special case of multiple regression analysis according to which one or more variables relate to others in the framework of a multidimensional crossing table. During this analysis, all homogrades are considered independent and any recess of the crossing table dependent [10]. Finally, in order to interpret the impacts on the model of optimum adjustment, we represent the data in the form of one or two-dimensional tables [14].

\section{RESULTS}

The city council of the municipality of Karditsa approved the new cleanness regulation with the decision number 220/1991 (Prefecture B 27882/18-12-91), dealing mainly with the maintenance of the city's cleanness, public health security, proper management of waste and solid waste (packaging, collection, removal, recycling, disposal), notification and compliance to the relevant public orders. However, the majority of the citizens $\left(57.7 \%, s_{p}=0.0252\right)$ has poor information of their obligations and rights, resulting from the regulation, whereas $20.5 \%\left(s_{p}=0.0206\right)$ is informed of the cleanness regulation. About $21.8 \%\left(s_{p}=0.0211\right)$ gave no answer.

The developed relations between the waste management carrier (Local Government Authorities, Waste Management Carrier), the operator of the production system on a daily basis (Public Cleanness Service, Association of Local Government Authorities, Private Enterprise, etc.) and the citizen as a user himself are expressed through four types of streams: (a) the authorization, that defines who authorizes whom and for what reason, (b) the service, that defines who renders service to whom, (c) the money, that refers to who pays to whom and (d) the rules, that describes who imposes the rules and to whom [2]. The citizens, as customers of the service, should have prior knowledge of the certain service in order that they are able to evaluate it. Therefore, an organized system for the management of urban solid waste should take efforts to inform the citizens. 
The declared information, by the residents, about waste management in the area of Karditsa cannot be characterized as successful. About $6 \%\left(s_{p}=0.0121\right)$ of the citizens answered that they are very well informed, $10.6 \%\left(s_{p}=0.0157\right)$ as well informed, $31.2 \%\left(s_{p}=0.0236\right)$ as sufficiently informed and $31.4 \%\left(s_{p}=0.0237\right)$ answered that they have poor information, whereas finally $20.8 \%$ $\left(s_{p}=0.0207\right)$ answered that they have no information at all.

Although the citizens state that the information they received is insufficient, it seems that the waste management system in the municipality of Karditsa is efficient, at least as far as the citizens' satisfaction is concerned. Therefore, $17.4 \%\left(s_{p}=0.0193\right)$ of the citizens found the rendered cleanness services by the municipality 'very satisfactory', whereas $5.5 \%\left(s_{p}=0.0116\right)$ described the services as 'disappointing'. Between these two extreme classes, there is a percentage of $58.4 \%$ $\left(s_{p}=0.0251\right)$ of the citizens who are satisfied with the rendered cleanness services, whereas $16.4 \%$ $\left(s_{p}=0.0190\right)$ find the services 'disappointing' and finally very few people, only $2.3 \%\left(s_{p}=0.0077\right)$ gave no response.

The provisional storage system (containers) makes the first impression on a citizen about the management system of urban solid waste and affects the whole performance of the system to a significant degree (expense, public health, social acceptance, etc.) [2]. We tried to evaluate the whole system using two elements; the distance one has to cover in order to reach the container and the waste collection frequency.

We asked the citizens to give an estimation of the located distance of the waste container from their house. About $36.4 \%\left(s_{p}=0.0245\right)$ of the population answered that the distance is less than $15 \mathrm{~m}, 42.9 \%\left(s_{p}=0.0253\right)$ answered $16-50 \mathrm{~m}$, and $15.6 \%\left(s_{p}=0.0185\right)$ noticed that the containers were at a distance between 51 and $100 \mathrm{~m}$ and finally 5.2\% $\left(s_{p}=0.0113\right)$ estimated that the distance was over $100 \mathrm{~m}$.

Consequently, the residents of the municipality ascertain that the distance of the waste containers from their doors is satisfactory, a fact that facilitates the waste disposal for them and therefore they seem to be satisfied with the rendered cleanness services of the municipality.

Although it is convenient for the citizens to have the containers near to the waste production point, the citizens do not wish to have these containers very close to their doors due to the annoying odor and the collection of strays (cats and dogs) and insects (flies and bees), especially during the summer. This results in the constant displacement of the containers. Approximately half of the citizens $\left(46.2 \%, s_{p}=0.0254\right)$ notice the displacement of the containers, whereas the other half $\left(47.3 \%, s_{p}=0.0255\right)$ did not notice such thing, and $6.5 \%\left(s_{p}=0.0126\right)$ gave no response. In particular, the displacement of the waste containers usually occurs in neighborhoods and not in the city center where the waste collection is more frequent and the distance between the containers is small.

This phenomenon is related to the viewpoint of the citizens about the containers and waste in general. A better image of the trash cans, the frequency of their collection in combination with informing the citizens that urban wastes are not infectious and the existence of adequately designed places for the wastes by the sidewalk could aid to the reduction of displacement of the containers and at the same time facilitate the job of the employees of the municipality's cleanness department. Furthermore, a schedule regulation for the hours during which the citizens should dispose their litter would also help immensely.

The waste collection frequency in the municipality of Karditsa can be described as 'satisfactory'. More specifically, $19.2 \%\left(s_{p}=0.0201\right)$ of the population responded that they are very much satisfied with the frequent waste collection, $60.3 \%\left(s_{p}=0.0250\right)$ responded that they are satisfied, $13.5 \%$ $\left(s_{p}=0.0174\right)$ seems to be disappointed and finally, a small percentage of $4.9 \%\left(s_{p}=0.0111\right)$ considers the collection frequency as 'very disappointing' and $2.1 \%\left(s_{p}=0.0073\right)$ gave no response. 
One-third of the citizens $\left(33 \%, s_{p}=0.0240\right)$ noted that the waste containers are being emptied 5 times per week, $15.8 \%\left(s_{p}=0.0186\right)$ stated 6 times per week, $15.1 \%\left(s_{p}=0.0183\right)$ stated 3 times per week and $14.3 \%\left(s_{p}=0.0179\right)$ stated 4 times per week. Furthermore, $3.1 \%\left(s_{p}=0.0089\right)$ believe that the containers are emptied once a week, $4.2 \%\left(s_{p}=0.0102\right)$ stated twice and finally $7.8 \%$ $\left(s_{p}=0.0137\right)$ stated 7 times per week.

The relevant authority of the municipality reports that besides the daily waste collection and emptying of containers, a nightly emptying also takes place during the weekends, when the amount of waste increases, and also during the day when an open market is held in the city center.

With reference to transportation hours of the domestic waste to the containers, a large percentage of the inhabitants $\left(32.5 \%, s_{p}=0.0239\right)$ states that this procedure takes place during morning hours. About $10.6 \%\left(s_{p}=0.0157\right)$ of the population claims that they dispose their waste at midday, $8.3 \%$ $\left(s_{p}=0.0141\right)$ in the afternoon, $24.7 \%\left(s_{p}=0.0220\right)$ in the evening and finally $23.9 \%\left(s_{p}=0.0218\right)$ at any time during the day.

In order to prevent annoyances, waste should not be placed too early at the collection points. The dust track should pass at predetermined hours and days, from the collection points; this would stimulate a positive response from the citizens. In this case, only $10 \%$ of the waste would be placed too early [1]. The information from the public and the accuracy in the time table of the waste collection results in the prompt waste collection from the containers which then usually remain empty. Certain municipalities in tourist areas allow waste throwing in certain hours, otherwise they fine their citizens.

Citizens were also questioned in whether they leave their garbage outside or by the trash-cans, when these are empty or if they ever captured themselves littering 'unconsciously' streets or public areas. Only $17.7 \%\left(s_{p}=0.0193\right)$ answered that they leave their waste outside the container, whereas the majority $80.5 \%\left(s_{p}=0.0201\right)$ refused to do such thing, and we did not receive any answer from $1.8 \%\left(s_{p}=0.0068\right)$.

This indicates that the inhabitants of Karditsa have accepted the changes promoted by the cleanness department of the municipality and that they are familiarized with the new methods, since they see that these changes improve their quality of life and the image of their city. Things are worse concerning the bad habit of littering 'unconsciously' streets and public areas; $54.5 \%\left(s_{p}=0.0254\right)$ responded negatively, whereas $43.9 \%\left(s_{p}=0.0253\right)$ responded positively, while a small percentage of $1.6 \%\left(s_{p}=0.0063\right)$ gave no response.

An effort to inform the citizens to place their waste inside the containers should take action. Since waste is not compressed adequately into the containers, we should urge them to try reducing the waste volume. Hence, with a minimal effort they will contribute to avoid 'overflowing' containers and to reduce the system's cost. In relation to the habit of littering streets, the educational culture should in the long term improve this antisocial behavior.

Finally, the citizens were asked to estimate the amount they pay as public fees for public cleanness and waste collection. The majority of the citizens $\left(48.1 \%\left(s_{p}=0.0255\right)\right)$ did not seem to have knowledge about the exact amount, whereas of the remaining citizens $10.6 \%\left(s_{p}=0.0157\right)$ estimated the amount to be $1 €$ per month, $11.7 \%\left(s_{p}=0.0164\right)$ estimated the amount to be between 1.1 and $2 €, 17.7 \%\left(s_{p}=0.0195\right)$ estimated the amount to be between 2.1 and $5 €, 5.5 \%\left(s_{p}=0.0116\right)$ estimated the amount to be between 5.1 and $10 €$ and $6.5 \%\left(s_{p}=0.0126\right)$ estimated the amount to be more than $10 €$.

Prior to the log-linear analysis application, we first examine the crossing table (Table 1) and observe that the only anticipated frequency lower than 5 is 3.4. Therefore, no anticipated frequency is lower than 1 and only one is lower than 5; hence, there exists no problem with low anticipated frequencies. We further observe that there is a disparity between the observed and the anticipated 
Table 1: Cross-tabulation of the three variables.

\begin{tabular}{|c|c|c|c|c|c|c|}
\hline \multirow{2}{*}{\multicolumn{4}{|c|}{ Containers' emptying per week }} & \multicolumn{2}{|c|}{ Cleanness services } & \multirow[b]{2}{*}{ Total } \\
\hline & & & & $\begin{array}{l}\text { Satisfied-very } \\
\text { satisfied }\end{array}$ & $\begin{array}{l}\text { Disappointed-very } \\
\text { disappointed }\end{array}$ & \\
\hline \multirow{6}{*}{$1-3$} & \multirow{4}{*}{$\begin{array}{l}\text { Distance }(\mathrm{m}) \text { from } \\
\text { waste containers }\end{array}$} & \multirow[t]{2}{*}{$\leq 50$} & Count & 56 & 17 & 73 \\
\hline & & & Expected count & 52.4 & 20.6 & 73.0 \\
\hline & & \multirow[t]{4}{*}{$>50$} & Count & 5 & 7 & 12 \\
\hline & & & Expected count & 8.6 & 3.4 & 12.0 \\
\hline & \multirow[t]{2}{*}{ Total } & & Count & 61 & 24 & 85 \\
\hline & & & Expected count & 61.0 & 24.0 & 85.0 \\
\hline \multirow[t]{6}{*}{$>3$} & \multirow{4}{*}{$\begin{array}{l}\text { Distance }(\mathrm{m}) \text { from } \\
\text { waste containers }\end{array}$} & \multirow[t]{2}{*}{$\leq 50$} & Count & 172 & 31 & 203 \\
\hline & & & Expected count & 164.3 & 38.7 & 203.0 \\
\hline & & \multirow[t]{2}{*}{$>50$} & Count & 40 & 19 & 59 \\
\hline & & & Expected count & 47.7 & 11.3 & 59.0 \\
\hline & \multirow{2}{*}{\multicolumn{2}{|c|}{ Total }} & Count & 212 & 50 & 262 \\
\hline & & & Expected count & 212.0 & 50.0 & 262.0 \\
\hline
\end{tabular}

Table 2: Nullity controls.

\begin{tabular}{|c|c|c|c|c|c|c|}
\hline$k$ & $d f$ & L.R. $\chi^{2}$ & Probability & Pearson $\chi^{2}$ & Probability & Iteration \\
\hline \multicolumn{7}{|c|}{ Tests that $k$-way and higher order effects are zero } \\
\hline 3 & 1 & 0.592 & 0.4416 & 0.589 & 0.4428 & 3 \\
\hline 2 & 4 & 19.415 & 0.0007 & 18.623 & 0.0009 & 2 \\
\hline 1 & 7 & 364.851 & 0.0000 & 483.086 & 0.0000 & 0 \\
\hline \multicolumn{7}{|c|}{ Tests that $k$-way effects are zero } \\
\hline 1 & 3 & 345.437 & 0.0000 & 464.463 & 0.0000 & 0 \\
\hline 2 & 3 & 18.823 & 0.0003 & 18.034 & 0.0004 & 0 \\
\hline 3 & 1 & 0.592 & 0.4416 & 0.589 & 0.4428 & 0 \\
\hline
\end{tabular}

$k$ : the number of effects being zero; $d f$ : degrees of freedom.

frequencies. This means that the assumption of full independency between these three criteria is incorrect.

The most adequate pattern for the application of the Hierarchical log-linear Analysis Program, after the term's removal of the third degree interaction, is the one that includes both main impacts and interactions of the variables divided by two. The interaction is eliminated according to three criteria, because $\chi^{2}$ of Pearson $=0.589$ is in effect with probability $(p)=0.042$ and $\chi^{2}$ probability ratio $=0.592$ with probability $(p)=0.043$. All of the above are confirmed by the test k-way and higher order effects are zero and the test k-way effects zero [12]. As shown in Table 2, there is no interaction according to three criteria, since the probability rate is $(p)=0.4428$. However, there is an interaction according to two criteria (since the probability $(p)<0.05$ ). In the three variable pairs (satisfaction-distance, satisfaction-number of times and distance-number of times) a significant 
Table 3: Frequencies observed and forecasted based on the hierarchical log-linear analysis.

\begin{tabular}{|c|c|c|c|c|}
\hline \multirow[b]{2}{*}{$\begin{array}{l}\text { Containers' emptying } \\
\text { per week }\end{array}$} & \multirow[b]{2}{*}{$\begin{array}{l}\text { Distance }(\mathrm{m}) \text { from } \\
\text { waste containers }\end{array}$} & & \multicolumn{2}{|c|}{ Cleanness services } \\
\hline & & & $\begin{array}{l}\text { Satisfied-very } \\
\text { satisfied }\end{array}$ & $\begin{array}{l}\text { Disappointed-very } \\
\text { disappointed }\end{array}$ \\
\hline \multirow[t]{4}{*}{$1-3$} & $\leq 50$ & Count & 56 & 17 \\
\hline & & Expected count & 54.9 & 18.1 \\
\hline & $>50$ & Count & 5 & 7 \\
\hline & & Expected count & 6.1 & 5.9 \\
\hline \multirow[t]{4}{*}{$>3$} & $\leq 50$ & Count & 172 & 31 \\
\hline & & Expected count & 173.0 & 30.0 \\
\hline & $>50$ & Count & 40 & 19 \\
\hline & & Expected count & 38.9 & 20.1 \\
\hline
\end{tabular}

Table 4: Cross-tabulation of the 'cleanness services' and 'distance (meter) from waste containers' variables.

\begin{tabular}{lllll}
\hline & & \multicolumn{2}{c}{$\begin{array}{c}\text { Distance }(\mathrm{m}) \text { from } \\
\text { waste containers }\end{array}$} & \\
\cline { 3 - 3 } & & $\leq 50$ & $>50$ & Total \\
\hline Cleanness services & Count & 246 & 46 & 292 \\
Satisfied-very & Expected count & 233.8 & 58.2 & 292.0 \\
satisfied & Residual & 12.2 & -12.2 & \\
& Count & 55 & 29 & 84 \\
Disappointed-very & Expected count & 67.2 & 16.8 & 84.0 \\
disappointed & Residual & -12.2 & 12.2 & \\
& Count & 301 & 75 & 376 \\
Total & Expected count & 301.0 & 75.0 & 376.0 \\
\hline
\end{tabular}

The total number of subjects (citizens) varies according to the missing values of the considered variables.

statistical interaction can be found. The observed and anticipated frequencies in accordance with the hierarchical log-linear analysis are cited in Table 3.

In order to interpret the impacts, we should first represent all data in the form of three twodimensional tables (cross-tables). With the aid of Table 4, we can see that the citizens who evaluate the cleanness services as 'satisfying' or 'very satisfying' live at a distance of $50 \mathrm{~m}$ or less from the trash cans, whereas those who state their disappointment are located at a distance of more than $50 \mathrm{~m}$. From Table 5, we can see that the citizens who evaluate the cleanness services as 'satisfying' or 'very satisfying' state that the containers are emptied more than 3 times per week, whereas waste collection 1-3 times per week leads the citizens to a negative evaluation. It is therefore obvious that the distance from the containers together with the waste collection frequency form a fundamental parameter that should be taken into consideration by the waste management department, to achieve a positive acceptance of the project by the citizens. 
Table 5: Cross-tabulation of the 'cleanness services' and 'containers' emptying times per week' variables.

\begin{tabular}{llccc}
\hline & & \multicolumn{2}{c}{$\begin{array}{c}\text { Containers' emptying times } \\
\text { per week }\end{array}$} & \\
\cline { 3 - 4 } Cleanness services & & $1-3$ & $>3$ & Total \\
\hline Satisfied-very & Count & 61 & 212 & 273 \\
satisfied & Expected count & 66.9 & 206.1 & 273.0 \\
& Residual & -5.9 & 5.9 & \\
Disappointed-very & Count & 24 & 50 & 74 \\
disappointed & Expected count & 18.1 & 55.9 & 74.0 \\
& Residual & 5.9 & 5.9 & \\
Total & Count & 85 & 262 & 347 \\
& Expected count & 85.0 & 262.0 & 347.0 \\
\hline
\end{tabular}

Table 6: Cross-tabulation of the 'distance (meter) from waste containers' and 'containers' emptying times per week' variables.

\begin{tabular}{lllll}
\hline & & \multicolumn{2}{c}{$\begin{array}{c}\text { Containers' emptying times } \\
\text { per week }\end{array}$} & \\
\cline { 3 - 4 } Distance $(\mathrm{m})$ from & waste containers & $1-3$ & $>3$ & Total \\
\hline$\leq 50$ & Count & 73 & 206 & 279 \\
& Expected count & 67.6 & 211.4 & 279.0 \\
& Residual & 5.4 & -5.4 & \\
$>50$ & Count & 13 & 63 & 76 \\
& Expected count & 18.4 & 57.6 & 76.0 \\
\multirow{3}{*}{ Total } & Residual & -5.4 & 5.4 & 355 \\
& Count & 86 & 269 & 355.0 \\
\hline
\end{tabular}

The third pair presented in Table 6 shows that when the distance from the waste containers is equal to or less than $50 \mathrm{~m}$, the citizens believe that the containers are emptied 1-3 times per week, in contrast to those who are at a distance of more than $50 \mathrm{~m}$ and believe that these are emptied more than 3 times per week. The citizens who live farther from the containers report that the containers are emptied more frequently because greater the distance, the fewer the containers that are filled quicker and therefore should be emptied more often. Here, we should also take into consideration the behavior of the working staff in dust tracks, who often do not empty containers that are not filled.

\section{DISCUSSION AND CONCLUSION}

Since 1991, the municipality of Karditsa has adopted a new cleanness regulation and probably more than half of its population has no knowledge of it at all. More than half of the residents state that they have poor or no information at all in relation to waste management in their area. It is therefore rather 
obvious that there is a lack in the citizens' information, a fact that reveals the necessity for establishing certain communication channels on behalf of the municipality towards the citizens, i.e. through informative brochures, advertisements in magazines, local radio and TV channels and public conversation. Besides the pursued aim, such an activity would show the citizens the interest and effort taken on behalf of the municipality to improve the quality of life within the city. Furthermore, the creation of a bidirectional communication channel (contact phone line) for the citizens to express their comments and complaints could lead to a constant improvement of the system.

Although the citizens seem to be insufficiently informed, they state that the waste management system is efficient in the municipality of Karditsa. Naturally, the contact and the knowledge of the citizens concerning the system for the management of urban solid waste refer mainly to the provisional storage system (containers) and waste collection system (time-table of the dust tracks). The distance the citizens need to cover to reach the containers and the frequency of the waste collection form the two main elements through which the system can be estimated.

Citizens who evaluate the cleanness services as 'satisfying' or 'very satisfying' live at a distance of $50 \mathrm{~m}$ or less from the waste containers, whereas those who state that they are 'disappointed' or 'very disappointed' live at a distance of more than $50 \mathrm{~m}$. Citizens who evaluate the cleanness services as 'satisfying' or 'very satisfying' state that the containers are emptied more than 3 times per week, whereas waste collection 1-3 times per week leads the citizens to a negative evaluation. It is therefore obvious that the distance from the containers together with the waste collection frequency form a fundamental parameter that should be taken into consideration by the waste management department. Thus, more than half of the citizens noted that the containers are being emptied at least 5 times per week, a fact that made them declare their satisfaction concerning the waste collection frequency.

Although it is convenient for the citizens to place the containers nearer to the waste production point, however, the citizens do not wish to have the containers near their doors and this often results in the displacement of the containers; only half of the citizens notice the displacement of the containers. Clean, freshly painted containers, which could close properly and will not overflow due to their frequent collection, proper coordination and programming for the hours during which the citizens will dispose their waste in them, together with the creation of properly designed places by the sidewalk for the containers, all these would immensely help to reduce this phenomenon and facilitate the work of the employees of the cleanness department.

The disposal of domestic waste into the containers takes place in different hours of the day. The majority (32.5\% of the citizens) disposes domestic waste during morning hours. The briefing of the public together with the precise consideration of the collection time table is expected to result in the prompt waste collection from the containers. As a consequence, the containers will remain empty longer and the feelings of annoyance by the neighbors will decrease.

Furthermore, when the distance of the citizens' residence is equal to or less than $50 \mathrm{~m}$ from the waste containers, the citizens believe that the containers are emptied 1-3 times per week, in contrast to those who live at a distance greater than $50 \mathrm{~m}$ and believe that these are emptied more than 3 times per week. Thus, greater the distance, the fewer the containers that are filled quicker and therefore should be emptied more often. Here, we should also take into consideration the behavior of the working staff in dust tracks, who often do not empty containers that are not filled. Waste leaving in winter is not a big issue; but in the summer, the high temperatures and increased fermentation of food residuals create undesirable odors.

The system management should therefore take into account the number of containers and combine the relevant accepted load of the containers with their collection frequency. More specifically, we conclude, indirectly through the citizens' viewpoint, that a certain form of planning occurs in 
the municipality of Karditsa, though the system could be further improved, for example, in the coordination of the containers' filling and emptying, control of the working staff, etc.

Certain citizens state that they leave their waste besides or outside the waste containers when these are empty, and more than half of the population confesses littering 'unconsciously' streets and public areas. An effort to inform the citizens to place their waste into the containers should take action, and since waste is not compressed adequately into the containers, we should urge them to try and reduce the waste volume. The citizens need to provide minimum effort to achieve a better situation of the containers which will lead to a significant reduction of the system's cost and an increase in its efficiency. Additional issues of antisocial behavior, such as littering of streets and public areas, need a long-term strategy for increasing educational culture.

Finally, half of the citizens are unaware of the amount they pay as public fees for public cleanness and waste collection. The question raised is how the citizens, as buyers of this service offered by the municipality, can demand its improvement when, at the same time, they are unaware of the amount they pay for it.

[1] Kollias, P.S., Waste, Athens, 1993.

[2] Panagiotakopoulos, D., Viable Management of Urban Solid Waste, Zigos Publications: Thessaloniki, 2002.

[3] Freese, F., Forest Sampling Data, (translation) ed. M.A. Karteris, Thessaloniki, 1984.

[4] Asteris, K.I., Forest Biometry, Vol. 1, Aristotle University of Thessaloniki: Thessaloniki, 1985.

[5] Matis, K.G., Forest Sampling, Thessaloniki, 1988.

[6] Damianos, X.X., Sampling Methodology: Techniques and Applications, 3rd printing, Aethra Publications, 1999.

[7] Kalamatianou, A.G., Social Statistics, One-Dimensional Analysis Methods, The Economic Publications: Athens, 2000.

[8] Philias, B., Pappas, P., Antonopoulos, M., Zarnaris, O., Maganara, I., Meimaris, M., Nikolakopoulos, H., Papachristos, E., Perantzakis, I., Samson, E. \& Psihogios, E., Introduction in the Methodology and Techniques of Social Researches, Gutenberg Social Library: Athens, 2000.

[9] Pagano, M. \& Gauvreau, K., Principles of Biostatistics, Ellin Publications, 2000.

[10] Siardos, G.K., Multivariable Statistical Analysis Methods. Part 1: Research in Variables' Relations, Ziti Publications: Thessaloniki, 1999.

[11] Tabachick, B.G. \& Fidell, L.S., Using Multivariate Statistics, 2nd edn, Harper and Row: New York, 1989.

[12] Norusis, M.J., SPSS Advanced Statistics 6.1, SPSS Inc.: Chicago, 1994.

[13] Frangos, X.K., Market Research Methodology and Data Analysis with the Application of the Statistical Package SPSS for Windows, Interbooks Publications: Athens, 2004.

[14] Howitt, D. \& Gramer, D., Statistics with SPSS 11 and Windows, Klidarithmos: Athens, 2003. 